\title{
Analysis of leukocyte transepithelial migration using an in vivo murine colonic loop model
}

\author{
Sven Flemming, Anny-Claude Luissint, Asma Nusrat, and Charles A. Parkos \\ Department of Pathology, University of Michigan, Ann Arbor, Michigan, USA
}

Molecular mechanisms that control leukocyte migration across the vascular endothelium (transendothelial migration; TEndoM) have been extensively characterized in vivo, but details of leukocyte transepithelial migration (TEPM) and its dysregulation (a pathologic feature of many mucosal diseases) are missing due to the lack of suitable animal models. Here, we describe a murine model that utilizes a vascularized proximal colonic segment (pcLoop) and enables quantitative studies of leukocyte trafficking across colonic epithelium. Consistent with previous in vitro studies, intraluminal injection of antibodies against integrin CD11b/CD18 reduced recruitment of polymorphonuclear neutrophils (PMN) into the lumen of pcLoops, and it increased subepithelial accumulation of PMN. We extended studies using the pcLoop to determine contributions of Junctional Adhesion Molecule-A (JAM-A, or F11R) in PMN TEpM and confirmed that mice with total loss of JAM-A or mice with intestinal epithelial selective loss of JAM-A had increased colonic permeability. Furthermore, there was reduced PMN migration into the colonic lumen that paralleled subepithelial accumulation of PMN in global-KO mice, as well as in intestinal epithelial-targeted JAM-A-deficient mice. These findings highlight a potentially novel role for JAM-A in regulating PMN TEpM in vivo and demonstrate utility of this model for identifying receptors that may be targeted in vivo to reduce pathologic intestinal inflammation.

Authorship note: SF and ACL contributed equally to this work.

Conflict of interest: The authors have declared that no conflict of interest exists.

License: Copyright 2018, American Society for Clinical Investigation.

Submitted: January 8, 2018 Accepted: September 11, 2018 Published: October 18, 2018

Reference information: JCI Insight. 2018;3(20): e99722. https://doi.org/10.1172/jici. insight.99722.

\section{Introduction}

Polymorphonuclear neutrophils (PMN) are first responders of the innate immune system and play a critical role in eradicating invading pathogens and antigens at epithelial surfaces. Despite the critical importance of PMN in host defense, dysregulated PMN recruitment across epithelial surfaces is linked to pathophysiology of a number of inflammatory diseases. In these circumstances, massive PMN transepithelial migration (TEpM) results in significant tissue damage and disease symptoms that is a characteristic feature of common debilitating conditions such as inflammatory bowel disease (IBD) $(1,2)$. Despite these observations, molecular mechanisms regulating PMN TEpM are far from being understood in contrast to an extensive body of knowledge on regulation of PMN migration across vascular endothelium (transendothelial migration, TEndoM). One major difference between the above 2 migratory processes lies in polarity of PMN migration that occurs in opposite directions with PMN transmigration from apical to basal aspect of endothelium in TEndoM and migration from basal to apical aspect of the epithelium in TEpM $(3,4)$. The vast majority of molecular details governing regulation of PMN TEpM have come from in vitro studies using transwell-based systems and suggest that PMN migrate across epithelia in a multistep process that involves surface molecules on leukocytes and epithelial specific counter receptors (4). Initial interactions in TEpM have been shown to involve PMN adhesion mediated by $\beta 2$-integrins (CD11b/CD18) to undetermined fucosylated counter ligands (5). After initial adhesion, PMN migrate along the paracellular space between epithelial cells in a CD47-dependent manner that results in transient remodeling of intercellular cell-to-cell junction complexes (paracellular migration) $(6,7)$. To reach the apical or luminal surface of the epithelium, PMN must traverse tight junctions (TJs) that serve to seal the paracellular space. In this regard, an epithelial TJ-associated transmembrane protein, Coxsackie and adenovirus receptor (CAR) has been described to mediate PMN TEpM through interactions with junctional adhesion molecule-L (JAM-L) that is expressed on PMN (8). Interestingly, another TJ-associated transmembrane protein JAM-A (F11R) has been reported to play an important role in the maintenance 
of epithelia and endothelia barrier function and regulate PMN migration across vascular endothelium in various models of inflammation (9-12); however, JAM-A contributions to the regulation of PMN TEpM in vivo have not been reported.

Since the vast majority of findings detailing regulation of PMN TEpM have been derived from transwell-based in vitro studies, there has been a long-standing need for complementary and confirmatory in vivo approaches. Several murine models that are widely used to study pathogenesis of IBD (13-15) are available, but none allow for quantitative and qualitative assessment of PMN TEpM in the colon, despite the central contribution of this process to IBD pathophysiology and other inflammatory disorders of mucosal surfaces. In addition, while there are a growing number of reports using live-cell imaging and 2-photon microscopy to track interactions of immune cells such as T cells, DCs with intestinal epithelium, or PMN migration out of blood vessels (TEndoM), none have investigated TEpM (16-22). Unlike subsets of T cells and DCs that naturally reside in the subepithelial or intraepithelial space in considerable abundance, PMNs are conspicuously absent and require a strong luminally directed chemotactic stimulus that represents a danger signal in order to drive egress of PMN from the microcirculation, through the subepithelial space and finally the epithelium. It is likely that technical challenges have limited progress on developing methods to specifically highlight intravital confocal imaging of PMN TEpM in the intestine.

Given the lack of in vivo approaches to study PMN TEpM, we developed an experimental model that enables quantitative and spatiotemporal studies of leukocyte trafficking across murine colonic mucosa in response to a luminally administered chemoattractants. Using a proximal colon loop (pcLoop) model described herein, quantification of PMN that have transmigrated into the colonic lumen or remain associated with the epithelium or subepithelial space (lamina propria-associated [LP-associated] PMN) is feasible using standard flow cytometric and immunohistochemical methods. We demonstrated the suitability of the pcLoop model to investigate the role of candidate adhesion molecules in regulating PMN TEpM by showing that intraluminal administration of neutralizing antibodies against leukocyte integrin CD11b/ $\mathrm{CD} 18(\alpha \mathrm{M} \beta 2 / \mathrm{Mac}-1)$ results in reduced PMN TEpM in response to the chemoattractant leukotriene $\mathrm{B}_{4}$ $\left(\mathrm{LTB}_{4}\right)$, confirming observations previously obtained using in vitro transwell-based cell migration systems (5). In addition, through the use of JAM-A global knockout mice $\left(F 11 r^{-/-}\right.$or $\left.\mathrm{Jam}_{-\mathrm{a}^{--}}\right)$and mice selectively lacking JAM-A in the intestinal epithelium (Villin-cre; Jam- $a^{f l / l}$ mice), we report that the epithelial expressed TJ-associated protein JAM-A not only plays an important role in regulating colonic epithelial barrier function, but serves as an important receptor that facilitates PMN TEpM.

\section{Results}

Establishment of the pcLoop model that enables rapid quantitative analysis of TEpM across the colonic mucosa. After crossing the vascular endothelium, PMN migrate across distinct compartments including sub-epithelial LP and a single layer of columnar epithelial cells to reach the colonic lumen. The pcLoop model described herein enables quantitative assessment of PMN at multiple stages of transmigration across intestinal mucosa including LP and epithelial associated PMN as well as those that have reached the colonic lumen. Absolute numbers of PMN were quantified using flow cytometry as well as visualization by microscopy after immunostaining (Figure 1A). Briefly, in this model, a $2 \mathrm{~cm}$ long segment of proximal colon was exteriorized without compromising blood supply after midline laparotomy in anesthetized mice as detailed in Figure 1B. The segment was cleared with Hank's Balanced Salt Solution (HBSS) to remove luminal contents followed by ligation of cut ends by suture. To induce PMN TEpM, a solution of $1 \mathrm{nM}$ $\mathrm{LTB}_{4}$ or the peptide chemoattractant $\mathrm{N}$-formyl-methionyl-leucyl-phenylalanine $(1 \mu \mathrm{M} \mathrm{N}$-formyl-methionyl-leucyl-phenylalanine [fMLF]) in $200 \mu 1$ of HBSS was administrated into the pcLoop lumen. After injection of chemoattractant, the loop was reinserted into the abdomen of the anesthetized mouse for 60 min, followed by excision of the ligated pcLoop and euthanasia. The contents of excised pcLoop were aspirated and combined with lumenal flushings containing $2 \mathrm{mM}$ EDTA in cold PBS to detach loosely adherent PMN from the mucosal surface. The luminal fraction was filtered through a $35 \mu \mathrm{m}$ cell strainer and the absolute numbers of PMN were quantified by flow cytometry using established PMN cell surface markers CD45, CD11b and Ly6G. Blood collections were used as positive controls for flow cytometric gating processes (Figure 1C). To confirm the accuracy of the flow cytometric strategy used to distinguish PMN in colonic luminal contents, experiments were performed with reporter mice expressing green fluorescence protein on myelomonocytic cells (LysM-eGFP mice) (23). The transmigrated PMN population was identified as either CD11b and Ly-6G/Gr1-double positive cells or GFP and Ly-6G/Gr1-double 


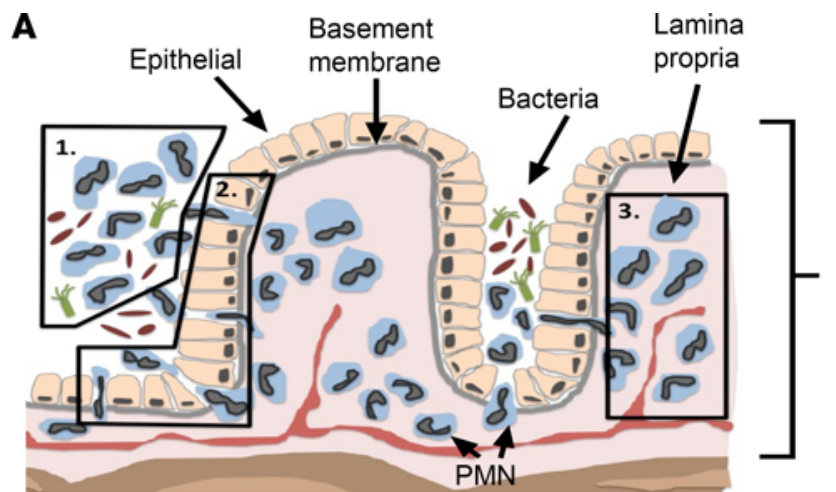

\section{Colonic crypts}

1. Luminal content

2. Epithelial-enriched fraction

3. Lamina propriaenriched fraction

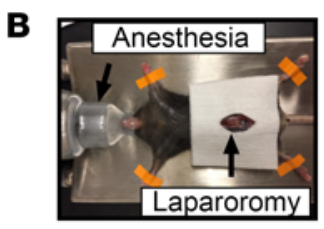

C Blood

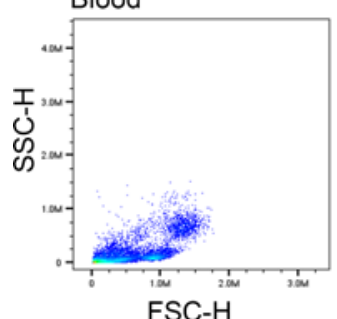

FSC-H Intestinal lumen

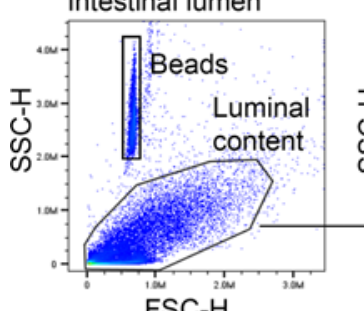

FSC-H
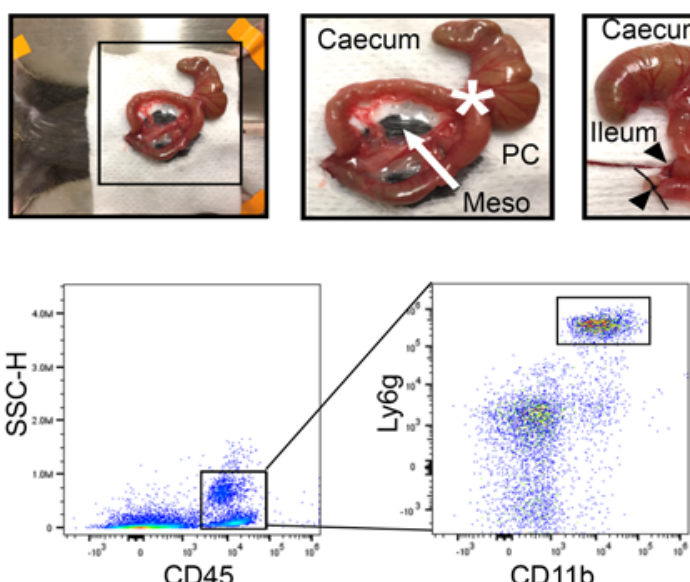

CD11b
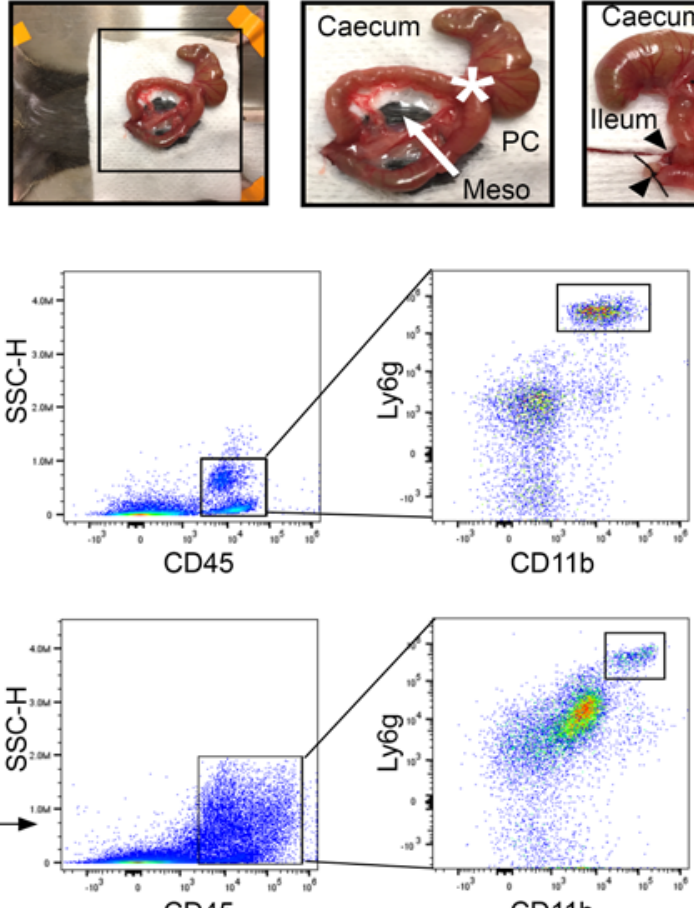

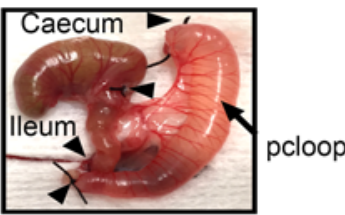

D

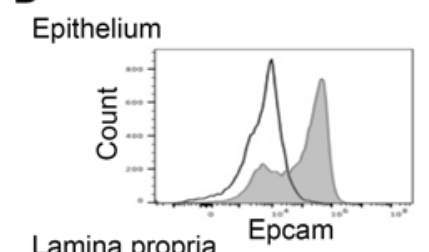

Lamina propria
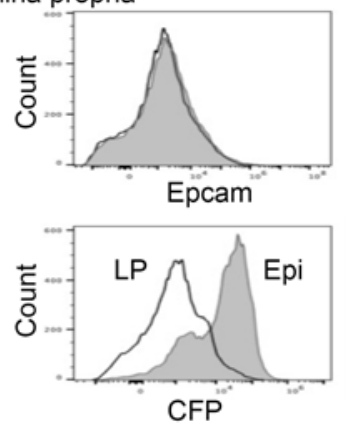

Figure 1. Proximal colon loop model. (A) Schematic presentation of pools of neutrophils (PMN) that are quantified in this study. Section 1 shows PMN migration into the colonic lumen, section 2 shows PMN interacting with the epithelial layer, and section 3 shows PMN in the lamina propria. Quantitative and qualitative analyses were performed by flow cytometry and IHC/immunofluorescence. (B) The proximal colon loop model (pcLoop): median laparotomy was performed in mice under isoflurane anesthesia on temperature controlled heating pads (left panel). Exteriorization of the caecum and a segment of proximal colon/PC (middle panels). Meso, mesocolon. Asterisk indicates ileo-caecal junction. PcLoop ( $2 \mathrm{~cm}$ length) after removal of feces and injection of a solution of chemoattractant to promote neutrophil recruitment into the colonic lumen (right panel). Arrowheads show 4 cut-ends ligation with silk suture. Then pcLoop was reinserted in the abdominal cavity for 1 hour. Immediately prior to euthanasia, the abdomen was reopened and pcLoop was excised for analysis. (C) Number of PMN (CD45+, Ly-6G/Gr1 ${ }^{+}$, and $\mathrm{CD}_{11 \mathrm{~b}}{ }^{+}$cells) quantified by flow cytometry using fluorescent counting beads. Circulating blood leukocytes were used as positive control for gating strategy. (D) Purity of the epithelial and lamina propria-enriched fractions using transgenic mice expressing the epithelial marker CFP-tagged E-cadherin fusion protein (B6.129P2(Cg)-Cdh1 $\left.{ }^{\text {tmicle }} /\right]$ mice). The epithelial marker EpCAM (gray) was detected in epithelium-enriched fractions and not lamina propria. Isotype control antibody is shown in white. The epithelial marker E-cadherin (CFP-tagged) was present in epithelium-enriched fractions (Epi/gray) but not in lamina propria-enriched fractions (LP/white).

positive cells (Supplemental Figure 1; supplemental material available online with this article; https:// doi.org/10.1172/jci.insight.99722DS1). After collection of luminal contents, residual pcLoop mucosa was processed and enzymatically digested to quantify epithelium-associated PMN and LP-associated PMN, respectively. To determine the purity of the epithelial and LP-enriched fractions, we used B6.129P2(Cg)-Cdh1 $1^{\mathrm{tm} 1 \mathrm{Cle}} / \mathrm{J}$ (E-cadherin-mCFP) mice that exhibit epithelial cells expressing E-cadherin tagged with Cyan fluorescent protein. As shown in Figure 1D, CFP-positive signal and epithelial cell adhesion molecule (EpCAM) were detected only in the epithelial fraction but not in the LP-enriched fraction, indicating high purity of each fractions following the described isolation method. 
A

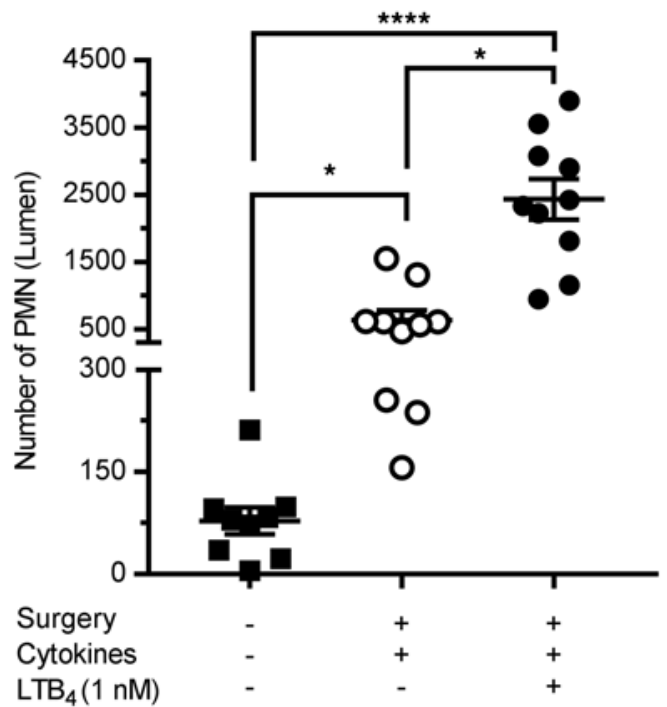

B Surgery + Cytokines

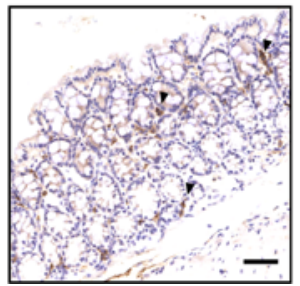

Surgery + Cytokines $+\mathrm{LTB}_{4}$

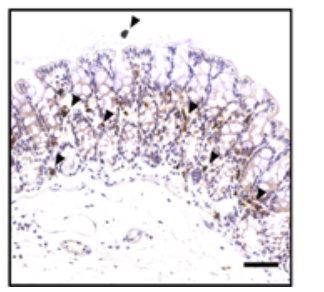

Figure 2. $\mathrm{LTB}_{4}$-dependent recruitment of PMN in the pcLoop. (A) Number of PMN recruited in the lumen of the pcLoop without chemoattractant (10 mice; white circles) or in the presence of $1 \mathrm{nM} \mathrm{LTB}_{4}$ (10 mice; black circles). Number of PMN present in the lumen of a segment of the proximal colon similar to the pcLoop at basal conditions (no surgery/9 mice; squares). Data are the mean $\pm \operatorname{SEM}(n=3$ independent experiments) and were analyzed by Kruskal-Wallis test with Dunn's multiple comparison test. ${ }^{*} P<0.05$, ${ }^{* * *} P<0.0001$. (B) Representative images of immunohistochemical staining of PMN (anti-Ly6C/Gr1 antibody) in the pcLoop of mice treated with cytokines only or in presence of LTB $_{4}$. $\mathrm{LTB}_{4}$ treatment resulted in an increased number of PMN in the pcLoop (arrowhead). Scale bars: $100 \mu \mathrm{m}$.

$\mathrm{LTB}_{4}$-dependent PMN recruitment into the pcLoop. Given that PMN are not abundant in the colonic subepithelial or intraepithelial space under normal conditions and require a danger signal for recruitment from the microcirculation, we used the well-accepted and potent physiologic PMN chemoattractant leukotriene $\mathrm{B}_{4}\left(\mathrm{LTB}_{4}\right)$ that has been shown to play a major role in PMN recruitment in a number of inflammatory conditions (24-29). By adding $\mathrm{LTB}_{4}$ to the lumen of the pcLoop, a transepithelial chemotactic gradient is created to stimulate PMN transmigration from the subepithelial space across the epithelium in a physiologically relevant basolateral to apical direction. In addition, to induce an inflammatory microenvironment that facilitates PMN transendothelial extravasation, mice were treated with proinflammatory cytokines TNF- $\alpha$ and IFN- $\gamma$ (100 ng/each) by i.p. injection 24 hours prior to pcLoop surgery (30-34). Such cytokine treatment resulted in a significantly increased recruitment of PMN into the colonic mucosa compared with untreated control mice not subjected to surgery (Figure 2A). Subsequent administration of $\operatorname{LTB}_{4}(1 \mathrm{nM})$ into the pcLoop lumen resulted in an abrupt increase in the number of transmigrated PMN, indicating $\mathrm{LTB}_{4}$-dependent PMN recruitment (Figure 2A). Enhanced infiltration of PMN into the colonic mucosa following stimulation by $\mathrm{LTB}_{4}$ when compared with cytokine-only treatment (no $\mathrm{LTB}_{4}$ ) was confirmed by immunohistochemical analyses (Figure 2B).

CD11b/CD18 is critical for PMN TEpM in the colon in vivo. The leukocyte-specific integrin CD11b/ CD18 $(\alpha \mathrm{M} \beta 2)$ has been shown to play a key role in regulating PMN TEpM in vitro by transwell-based systems, but in vivo methods of TEpM have not been available to confirm these findings in the intestine (5). We thus investigated the role of CD11b/CD18 in TEpM in vivo using the pcLoop model by administration of well-characterized function blocking mAbs against CD11b/CD18. Antibodies were injected in the lumen of the pcLoop 30 minutes prior administration of chemoattractant. Analyses of PMN migration after 60 minutes revealed significantly reduced transmigrated PMN in anti-CD11b/CD18-treated pcLoop compared with isotype control $\mathrm{mAbs}$, consistent with previously reported in vitro findings (Figure $3 \mathrm{~A}$ ). In addition, examination of PMN recruitment in epithelial- and LP-enriched fractions revealed increased numbers of PMN in LP fraction after blocking CD11b/CD18 (Figure 3B) with no change in epithelial-associated fractions. These findings indicate that intraluminal injection of $\mathrm{CD} 11 \mathrm{~b} / \mathrm{CD} 18$ did not prevent PMN to be efficiently recruited in the subepithelial/LP compartment of the pcLoop in response of $\mathrm{LTB}_{4}$; however, PMN migration into the pcLoop lumen was significantly impaired. 
A

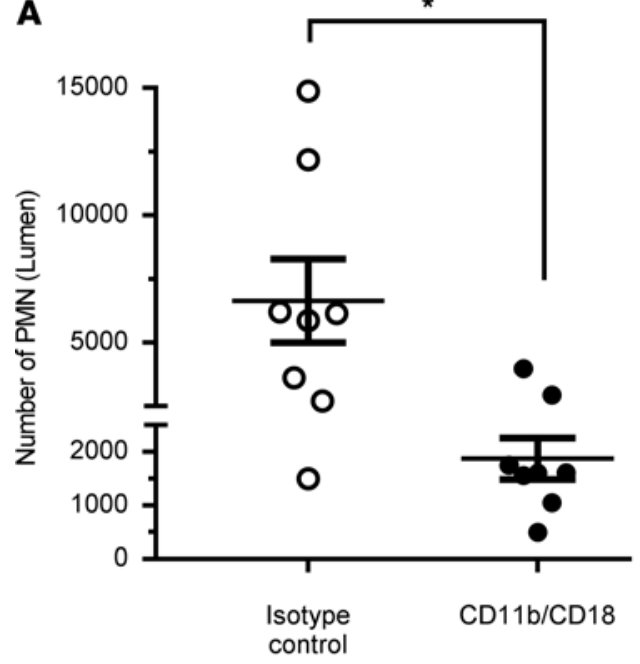

B

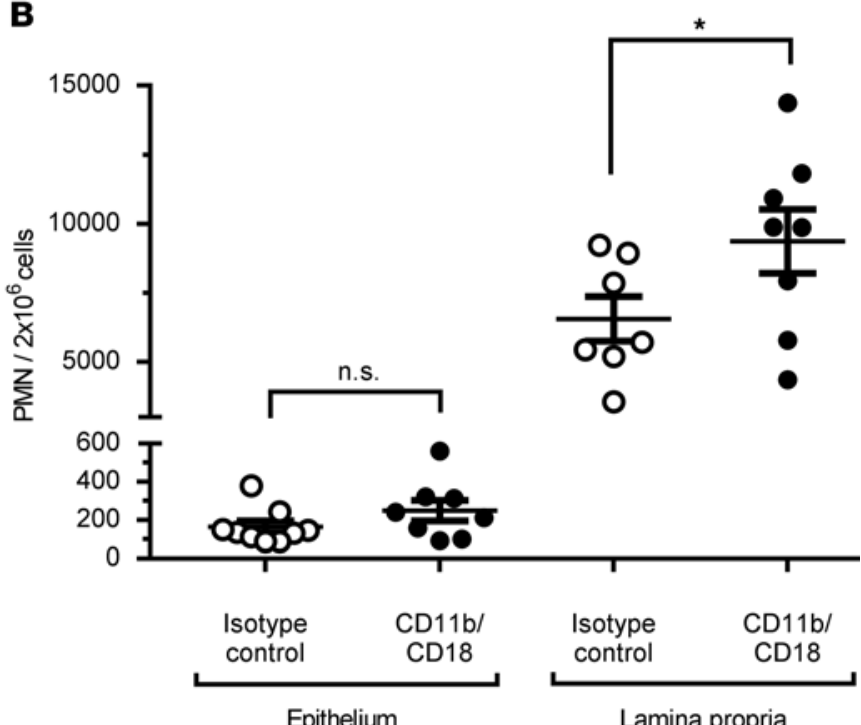

D

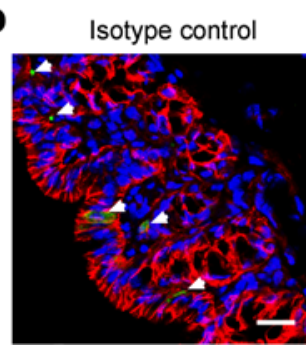

$\mathrm{CD} 11 \mathrm{~b} / \mathrm{CD} 18$

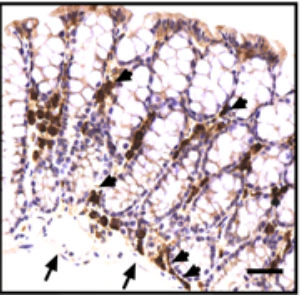

Isotype control

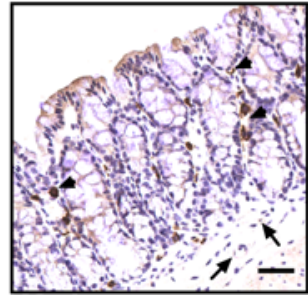

Figure 3. PMN TEPM in pcLoop model in response to $\mathrm{LTB}_{4}$ is dependent on leukocyte integrin CD11b/CD18. (A) Number of PMN recruited in lumen of the pcLoop after luminal injection of function blocking mAbs against CD11b/CD18 (8 mice; black circles) versus isotype control mAbs (8 mice; white circles). Data are the mean \pm SEM ( $n=2$ independent experiments) and were analyzed by 2 -tailed Student's $t$ test. ${ }^{*} P<0.05$. (B) Number of PMN in the lamina propriaand epithelium-enriched fractions after luminal injection of CD11b/CD18 mAbs (black circles) or isotype control mAbs (white circles). Epithelium-enriched fraction: Isotype control mAbs (9 mice) and CD11b/CD18 mAbs (8 mice). Lamina propria-enriched fraction: Isotype control mAbs (7 mice) and CD11b/CD18 mAbs (8 mice). Data are means \pm SEM; $n=3$ independent experiments. ${ }^{*} P<0.05$, 1-way ANOVA followed by Bonferroni's post-hoc multiple comparison tests. (C and $\mathbf{D})$ Representative images of Ly-6C/Gr1 (PMN) immunostaining of the pcLoop from mice treated with isotype control mAbs or CD11b/CD18 mAbs. (C) DAB IHC. Scale bars: $100 \mu \mathrm{m}$. (D) Immunofluorescence staining and confocal images. PMN (green), nuclei/DAPI (blue), epithelium/E-cadherin (red). Scale bars: $20 \mu \mathrm{m}$. Arrowheads, PMN; arrows, blood vessels.

Immunohistochemical analyses confirmed a subepithelial accumulation of PMN in animals treated with antibodies against CD11b/CD18 compared with those treated with isotype controls (Figure 3C). Immunofluorescence microscopic staining of PMN in the pcLoop following TEpM revealed many PMN in close apposition to the basal aspect of the intestinal epithelium after inhibition of CD11b/CD18 in comparison with controls (Figure 3D). These are the first observations to demonstrate a critical role of $\mathrm{CD} 11 \mathrm{~b} / \mathrm{CD} 18$ in regulating TEpM of PMN across the colonic epithelium in vivo.

Loss of JAM-A on epithelial cells diminishes PMN TEpM. We next used the pcLoop model to investigate the role of a TJ-associated adhesion molecule termed JAM-A on TEpM in vivo. JAM-A is a transmembrane protein abundantly expressed at the apical most components of the cell-to-cell junctional complexes in endothelia and epithelia. JAM-A has been shown to play a key role in regulating epithelial permeability and homeostasis $(11,35)$. Furthermore, given reports of JAM-A-mediated facilitation of leukocyte transendothelial migration, we reasoned that JAM-A might also play a role in regulating PMN TEpM $(36,37)$. Consistent with a previous report, analysis of JAM-A-global KO mice $\left(\mathrm{Jam}^{-a^{-/}}\right)$ revealed enhanced intestinal permeability to $4 \mathrm{kDa}$ FITC-dextran (Figure 4A) and increased numbers of LP-associated PMN under basal conditions (38) (Figure 4B). We then performed PMN TEpM experiments with the pcLoop in Jam- $a^{-1-}$ mice and observed significantly decreased TEpM in response to $\mathrm{LTB}_{4}$ compared with control mice (Figure 4C). Interestingly, analysis of the epithelium-enriched fraction revealed no significant difference in the number of PMN associated with the epithelium 


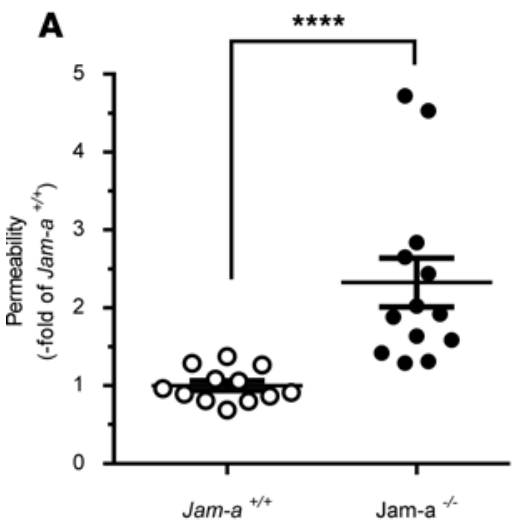

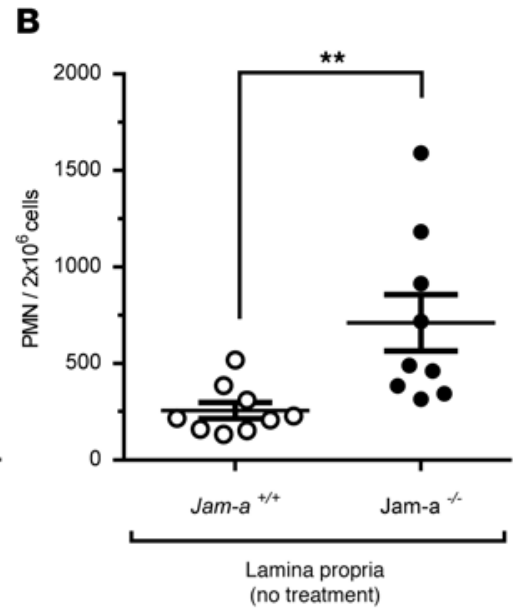

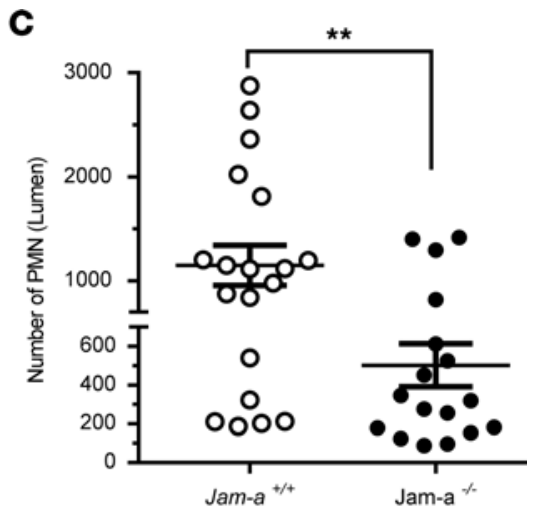

D

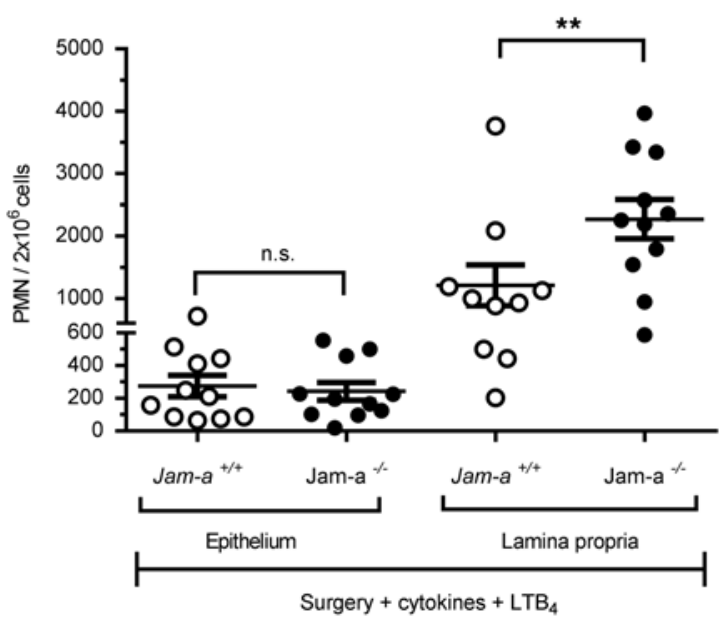

E

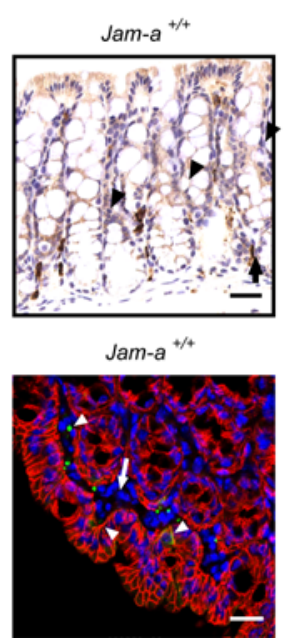

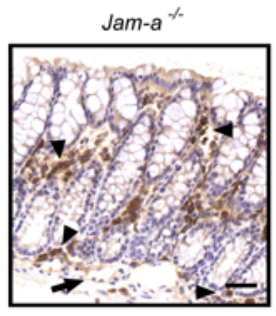

Jam-a

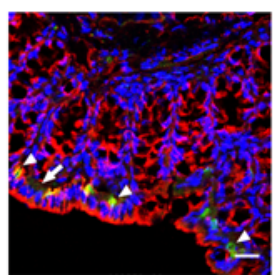

Figure 4. JAM-A facilitates PMN recruitment across colonic epithelium in vivo. (A) Loss of JAM-A (Jam- $a^{-/-}$) resulted in increased colonic epithelial paracellular permeability to $4 \mathrm{kDa}$ FITC dextran. Jam-- ${ }^{-/-}$(13 mice; black circles) were compared with Jam- $a^{+/+}$(12 mice; white circles). FITC dextran (1 $\mathrm{mg} / \mathrm{ml}$ ) was administrated into pcLoop lumen in the same buffers as LTB, and fluorescence was measured in serum after cardiac puncture after 60 minutes (end point of PMN TEpM assay). Data are expressed as means \pm SEM; $n=3$ independent experiments. ${ }^{* * * *} P<0.0001 ;$ Mann-Whitney $U$ test. (B) Number of PMN in the lamina propria-enriched fraction of a segment of proximal colon (similar to pcLoop) at basal conditions (no surgery) in $\mathrm{Jam}-a^{+/+}$(9 mice; white circles) and Jam- $a^{-/-}$mice (9 mice; black circles). Data are means $\pm \mathrm{SEM} ; n=3$ independent experiments. ${ }^{* *} P \leq 0.01,2$-tailed Student's $t$ test. (C) Number of PMN in the pcLoop lumen in response to $1 \mathrm{nM} \mathrm{LTB}{ }_{4}$ in Jam- $a^{+/+}$(19 mice; white circles) versus Jam- $a^{-/-}$mice (17 mice; black circles). Data are means \pm SEM; $n=4$ independent experiments. ${ }^{* *} P<0.01,2$-tailed Student's $t$ test. (D) Number of PMN recruited in the lamina propria and epithelium fractions of the pcLoop in response to $1 \mathrm{nM} \mathrm{LTB}_{4}$ in control Jam- $a^{+/+}$(white circles) and Jam-a $a^{-/-}$mice (black circles). Epithelium-enriched fraction: Jam- $a^{+/+}$(11 mice) and Jam- $a^{-/-}$(11 mice). Lamina propria-enriched fraction: Jam- $a^{+/+}$(10 mice) and Jam- $a^{-/-}$(11 mice). Data are means $\pm \mathrm{SEM} ; n=3$ independent experiments. ${ }^{* *} P \leq 0.01$, 1-way ANOVA followed by Bonferroni's post-hoc multiple comparison tests. (E and $\mathbf{F}$ ) Representative images of immunostaining for Ly-6G/Gr1 (PMN) in the pcLoop. (E) DAB IHC. Scale bars: $100 \mu \mathrm{m}$. (F) Immunofluorescence staining and confocal images. PMN (green), nuclei/DAPI (blue), epithelium/E-cadherin (red). Scale bars: $20 \mu \mathrm{m}$. Arrowheads, PMN; arrows, blood vessels.

between $\mathrm{Jam}-\mathrm{a}^{-/-}$and control $\mathrm{Jam}-\mathrm{a}^{+/+}$mice. However, there was a significant increased number of LP-associated PMN in Jam- $a^{-/-}$mice compared with controls. Noteworthy, there was increased infiltra-

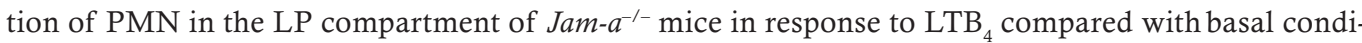
tions (Figure 4, B-D), consistent with large-scale recruitment of PMN into the colonic mucosa in both control and Jam-a $a^{-/-}$mice. However, less PMN were isolated in the pcLoop lumen of Jam- $a^{-/-}$animals, consistent with trapping of PMN in the subepithelial compartment (Figure 4, C and D). Immunohistochemical and immunofluorescence analyses supported these results, demonstrating an increased number of PMN in the pcLoop of Jam- $a^{-/-}$mice compared with control animals in response to $\mathrm{LTB}_{4}$ (Figure 4, E and F). In Jam- $a^{-/-}$mice, PMN were detected in close apposition to the basal aspect of the epithelium. However, in contrast to CD11b/CD18 inhibition, no PMN clusters were observed in $\mathrm{Jam}_{-a^{-/-}}$mice (Figure 3D). 
Since JAM-A is expressed on many cells (including endothelial and epithelial cells, as well as leukocytes), we generated epithelial-targeted JAM-A-deficient mice (Villin-cre; Jam-a $a^{f l / f l}$ mice) to investigate the epithelial-specific contributions of JAM-A in PMN TEpM. We confirmed epithelial-targeted loss of JAM-A in the colon by immunofluorescence staining (Figure 5A). Similar to Jam- $a^{-/}$mice, selective loss of intestinal epithelial JAM-A resulted in increased intestinal permeability to $4 \mathrm{kDa}$ FITC-dextran (Figure 5B) and enhanced LP-associated PMN under basal conditions (Figure 5C), confirming an important role for epithelial JAM-A in the regulation of intestinal barrier function and homeostasis. In addition, we observed significantly reduced recruitment of PMN into the colonic lumen in Villin-cre; Jam-a $a^{\text {flfl }}$ mice (Figure 5D) that was associated with an accumulation of PMN in LP-enriched fractions (Figure 5E). Since endothelia and leukocytes still express JAM-A in Villin-cre; Jam- $a^{f l f l}$ mice, these observations indicate that JAM-A expression on intestinal epithelial cells (IECs) is needed for efficient TEpM of PMN in vivo. The increased numbers of PMN present in the LP in Villin-cre; Jam-alflf in response to $\mathrm{LTB}_{4}$ (Figures 5, C-E) indicates efficient recruitment of PMN into pcLoop mucosa; however, trafficking of PMN across the colonic epithelium is impaired despite conditions of enhanced paracellular permeability. Collectively, these findings support a new key role for epithelial-expressed JAM-A in facilitating PMN TEpM beside its role in the maintenance of mucosal barrier function.

Given that JAM-A deficiency resulted in enhanced intestinal permeability (Figures 4A and Figure 5B), we performed experiments to exclude the possibility that the observed decrease in PMN TEpM was not simply due to cell desensitization and/or loss of chemotactic effect secondary to a leaky barrier and increased exposure to $\mathrm{LTB}_{4}$. Taking advantage of the known effects of high-dose TNF- $\alpha$ exposure on increasing intestinal permeability (39), we performed PMN TEpM experiments in pcLoops of mice that had been treated with high doses of cytokines. While there was no significant increase in permeability in mice pretreated with $100 \mathrm{ng}$ each of TNF- $\alpha$ and IFN- $\gamma$, pretreatment with $1 \mu \mathrm{g}$ of each cytokine resulted in significantly increased permeability to $4 \mathrm{kDa}$ FITC dextran that reached levels similar to those observed in JAM-A-deficient mice (Figures 6A and Figure 5B). Importantly, as shown in Figure 6B, we observed that PMN TEpM was not reduced but, in fact, was significantly increased in mice with enhanced intestinal permeability caused by exposure to high doses of proinflammatory cytokines when compared with treatment with low doses of cytokines (100 ng/each). These observations lend further support for a direct role of epithelial-expressed JAM-A in regulating TEpM that is independent of the role in regulating epithelial barrier function.

\section{Discussion}

In this report, we describe a unique in vivo model designed to study TEpM of PMN in the colon under acute inflammatory conditions. Since the exteriorized pcLoop is fully vascularized, it offers advantages over ex vivo chamber-based approaches, as well as new opportunities for physiological studies aimed at elucidating molecular mechanisms regulating acute PMN recruitment to the colonic mucosa and PMN TEpM in living mice. Furthermore, the pcLoop model enables quantitative assessment of PMN that have migrated across the epithelium into the colon lumen, as well as populations of PMN that remain associated with the epithelium or within the subepithelial space.

Numerous experimental murine models have been generated to mimic various aspects of human IBD and infectious enterocolitis. In particular, dextran sulfate sodium-induced (DSS-induced) colitis, IL-10KO-induced colitis, Citrobacter rodentium, Salmonella typhimurium, and other models have contributed to a better understanding of disease but are not good models for mechanistically investigating acute trafficking of PMN into the intestinal epithelium since the observed inflammatory responses take place over a course of days rather than minutes (13-15). Others have also reported the use of intestinal loops in a variety of animals to study intestinal physiology and antigen-presenting cell function; however, there are no studies employing exteriorized colon loops investigating mechanisms of leukocyte TEpM, despite the strong association of disease pathophysiology in IBD with PMN TEpM $(13,16,40,41)$. The pcLoop model fills this gap by allowing for assessment of PMN recruitment over a time course of minutes and, thus, provides unique opportunities for mechanistic studies. While small intestine loops are easier to mobilize and exteriorize, the pcLoop is easily performed after appropriate training and provides the additional advantage of appropriate modeling of inflammatory processes that are localized to the colon, such as ulcerative colitis.

In this report, we show the utility of the pcLoop model in characterizing molecular events regulating PMN TEpM in response to luminal administration of $\mathrm{LTB}_{4}$. We verified the utility of this model for assessing TEpM in response to other chemoattractants by performing the pcLoop experiments with 
A

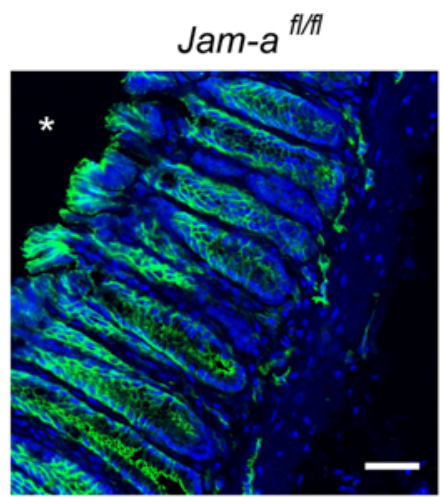

B

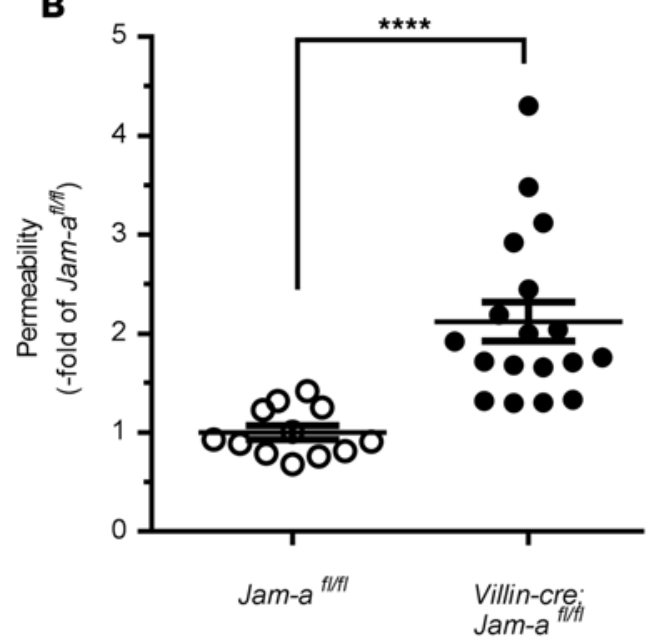

D

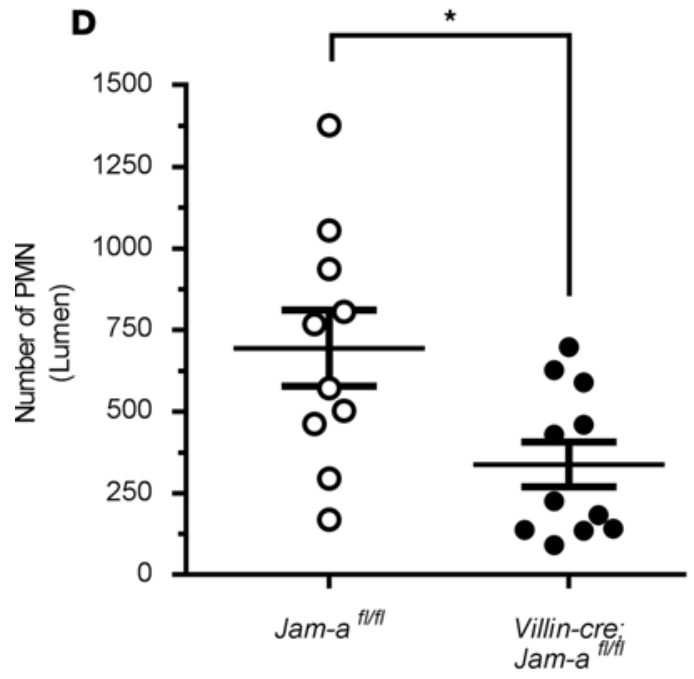

Villin-cre; Jam-a ${ }^{\text {fl/f }}$
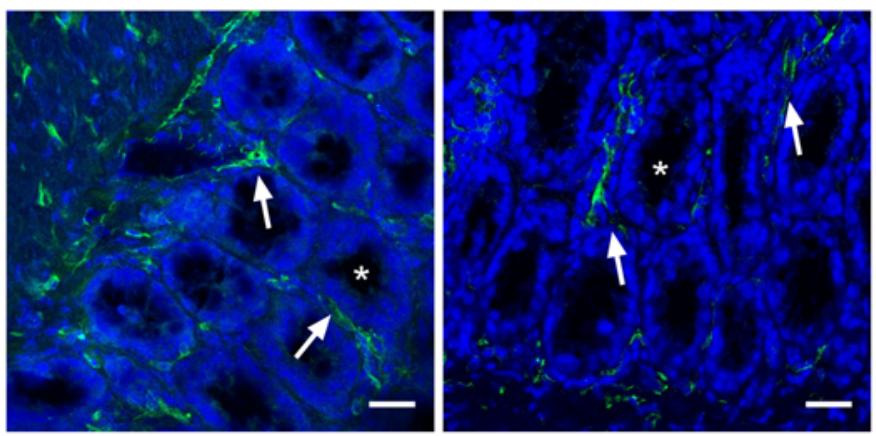

C

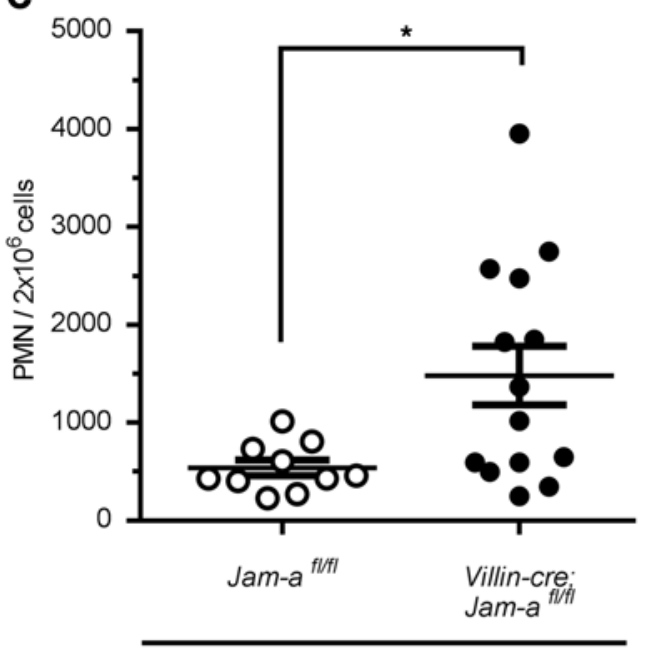

Lamina propria

( no treatment )

E

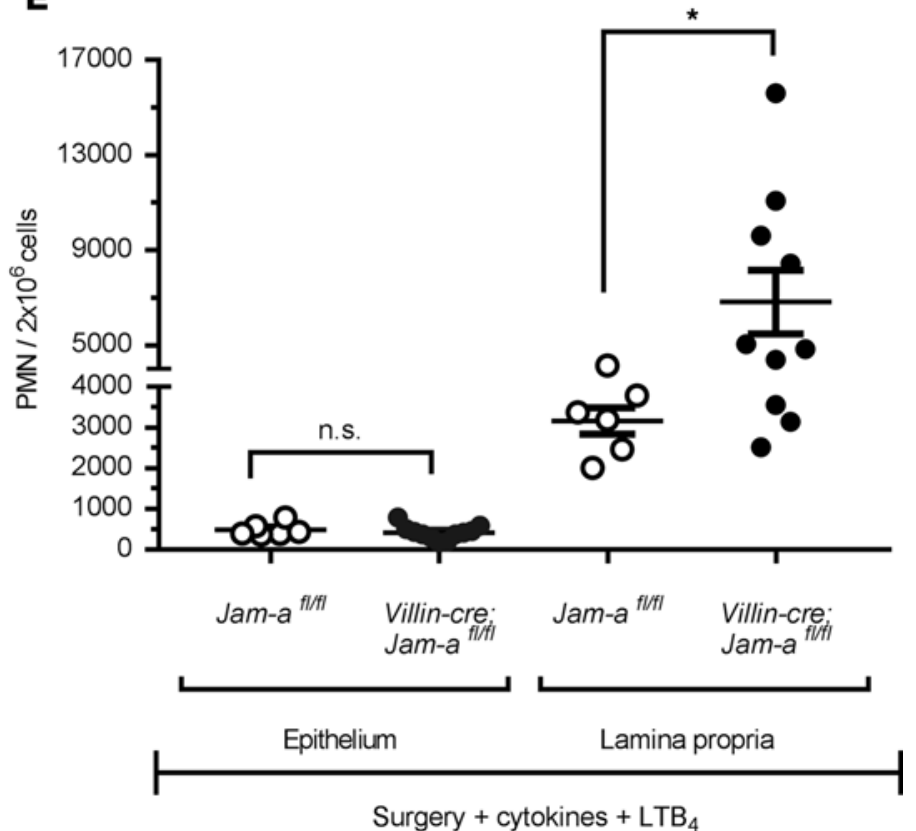

Figure 5. Selective loss of JAM-A on intestinal epithelial cells results in increased epithelial permeability and decreased PMN TEpM. (A) Immunofluorescence staining showing selective loss of JAM-A (green) on intestinal epithelial cells in Villin-cre; Jam-afl/fl mice compared with control Jam- $a^{f / / f l}$ mice. JAM-A is still present in the lamina propria compartment (arrows) including endothelial cells and leukocytes. Cell nuclei (Hoechst; blue). Asterisk represents intestinal lumen. Scale bars: $100 \mu \mathrm{m}$. (B) Permeability assay to $4 \mathrm{kDa}$ FITC dextran $(1 \mathrm{mg} / \mathrm{ml})$ showed significantly increased intestinal 
paracellular permeability in Villin-cre; Jam- $a^{f / f l}$ (18 mice; black circles) compared with control (Jam- $a^{f / f f}$, white circles: 12 mice). Data are means \pm SEM; $n$ $=4$ independent experiments. ${ }^{* * *} P \leq 0.0001$; Mann-Whitney $U$ test. (C) Number of PMN present in the lamina propria-enriched fraction of a segment of proximal colon (similar to pcLoop) under basal conditions (no surgery) in Jam-a $a^{f / f}$ mice (10 mice; white circles) and Villin-cre; Jam-a $a^{f / f l}$ mice (14 mice; black circles). Data are means \pm SEM; $n=3$ independent experiments. ${ }^{* *} P<0.01 ; 2$-tailed Student's $t$ test. (D) Number of PMN in the pcLoop lumen in response to $1 \mathrm{nM} \mathrm{LTB}$ in Villin-cre; Jam- $a^{f / f l}$ mice (11 mice; black circles) and Jam-a $a^{f / f l}$ mice (10 mice; white circles). Data are means $\pm S E M ; ~ n=3$ independent experiments. ${ }^{*} P<0.05$; 2-tailed Student's $t$ test. (E) Number of PMN in the lamina propria and epithelium fractions in pcLoop in control Jam- $a^{f / / f l}$ (white circles) and Villin-cre; Jam-a $a^{f / f l}$ mice (black circles). Epithelium-enriched fraction: Jam- $a^{f / f l}$ (6 mice) and Villin-cre; Jam-a $a^{f / f l}$ mice (11 mice). Lamina propria-enriched fraction: Jam- $a^{f / f l}\left(6\right.$ mice) and Villin-cre; Jam- $a^{f / f l}$ mice (10 mice). Data are means $\pm \mathrm{SEM} ; n=2$ independent experiments. ${ }^{*} P<0.05$; 1-way ANOVA followed by Bonferroni's post-hoc multiple comparison tests.

the chemotactic peptide fMLF, demonstrating significant recruitment of PMN into the colonic lumen (Supplemental Figure 3).

We also demonstrate that intraluminal administration of neutralizing antibodies against the leukocyte integrin CD11b/CD18 result in significant inhibition of $\mathrm{LTB}_{4}$-stimulated PMN TEpM and arrest of PMN in close apposition to the basal aspect of the colonic epithelium. These findings confirm previous results that have, until now, only been assessed with in vitro systems using cultured cells and transwell-based models (5). While CD11b/CD18 is well appreciated to play a major role in firm adhesion and PMN diapedesis across the vascular endothelium, we did not observe massive accumulation of PMN inside blood vessels in the colon, suggesting that injection of the neutralizing antibodies into the lumen of the pcLoop did not diffuse into the vascular compartment and prevent PMN interactions with endothelial cells. These observations are, thus, consistent with impaired CD11b/ CD18-dependent PMN TEpM. While basally expressed epithelial counter-receptors for CD11b/CD18 remain unknown, a previous in vitro study revealed that fucosylated epithelial proteoglycans mediate initial PMN adhesion to the basal aspect of human intestinal epithelial cells (42). Interestingly, despite an abundance of crypt-associated PMN observed in human diseases associated with massive TEpM, microscopic analyses of pcLoop segments after TEpM did not reveal areas of preferential PMN migration across crypt versus surface epithelium. Furthermore, since TEpM of PMN was not totally abolished after neutralization of $\mathrm{CD} 11 \mathrm{~b} / \mathrm{CD} 18$, these findings raise the possibility that PMN receptors other than $\mathrm{CD} 11 \mathrm{~b} / \mathrm{CD} 18$ may mediate early adhesive events in TEpM. Given the positive inhibitory effects observed with intraluminal injection of anti-CD11b/CD18, the pcLoop model can be successfully employed to study the local effects of compounds intraluminally administered on the intestinal mucosal homeostasis and immune responses.

With the pcLoop, we also provide the first evidence to our knowledge linking the TJ-associated protein JAM-A in directly regulating PMN TEpM. To date, none of the antibodies that have been reported to block JAM-A barrier function were described to prevent PMN TEpM in vitro. The lack of inhibition may be due to the fact that a majority of extracellular-targeting JAM-A antibodies bind to the membrane distal Ig-like domain, which has been shown to be necessary for regulating barrier function but not for binding to PMN. There are in vitro reports of JAM-A mediating binding to LFA-1 on leukocytes via the membrane proximal Ig loop $(43,44)$; however, LFA-1 does not play a role in regulating PMN TEpM $(5,45)$. We thus used the loop model with JAM-A-deficient mice to definitively assess the role of JAM-A in TEpM in vivo. We demonstrated enhanced intestinal permeability in the pcLoop in both Jam- $a^{-/-}$and Villin-cre; Jam-a $a^{f / f l}$ mice, confirming a major role for epithelial-expressed JAM-A in the maintenance of colonic barrier function. Interestingly, while Jam- $a^{-1-}$ and Villin-cre; Jam- $a^{f / f l}$ mice exhibited an enhanced PMN infiltration in the LP-enriched fraction, TEpM of PMN was significantly decreased in response to $\mathrm{LTB}_{4}$ in the pcLoop. Immunohistochemical and immunofluorescence analyses were consistent with $\mathrm{LTB}_{4}$-driven accumulation of PMN in the subepithelial space in Jam- $a^{-/-}$mice. The similarity of results in epithelial-targeted JAM-A-deficient mice with those in total KO suggest that leukocyte expressed JAM-A does not play a significant role in $\mathrm{LTB}_{4}$-stimulated migration of PMN in the intestine. Interestingly, there are 2 studies that have reported JAM-A-null PMN exhibited impaired chemotaxis $(46,47)$; however, our findings are consistent with JAM-A-mediated effects on leukocyte recruitment being stimulus specific (37). The approach in this study employed in vivo use of Villin-cre; $J a m-a^{f l / f l}$ mice that selectively lack JAM-A on intestinal epithelial cells, thereby confirming specific contributions of epithelial-expressed JAM-A in mediating PMN TEpM. Precise mechanisms by which epithelial-expressed JAM-A regulate PMN TEpM remains to be investigated in future studies. It is likely that epithelial JAM-A that is localized at TJ might play a major role in the signaling pathways that are 
A

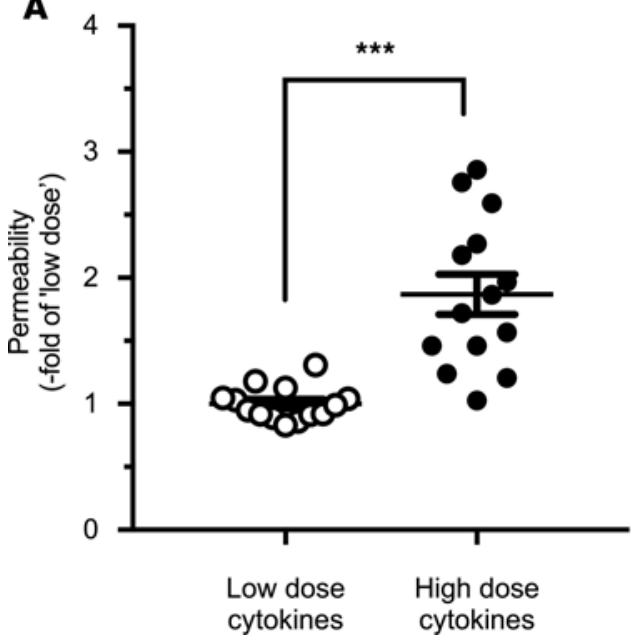

$\mathbf{B}$

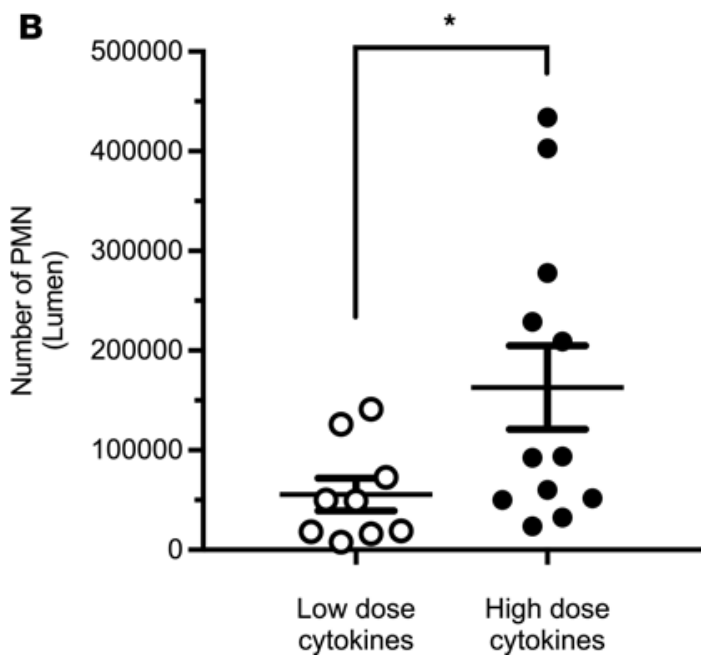

Figure 6. Enhanced paracellular permeability following treatment with a high dose of proinflammatory cytokines results in enhanced PMN TEPM. (A) I.p. injections of TNF- $\alpha$ and IFN- $\gamma$ at low dose (100 ng each/14 mice; white circles) or high dose (1 mg each/14 mice; black circles) 24 hours prior to generation of the pcLoop model. Permeability assay using $4 \mathrm{kDa}$ FITC dextran revealed a significant increase in the pcLoop paracellular permeability in animals treated with a high dose of cytokines. Data are means $\pm \mathrm{SEM} ; n=3$ independent experiments. ${ }^{* *} P<0.001 ; 2$-tailed Student's $t$ test (B) High-dose cytokine-treated mice showed enhanced PMN TEpM (12 mice; black circles) compared with low-dose cytokine-treated mice ( 9 animals; white circles). Data are means $\pm S E M ; n=3$ independent experiments. ${ }^{*} P=0.03 ; 2$-tailed Student's $t$ test.

necessary for efficient opening of the cell-to-cell junction allowing PMN migration through adjacent IECs. In addition, given reported binding properties of JAM-A with itself and integrins, we predict that homophilic JAM-A interactions in trans between migrating PMN and epithelial cells or heterophilic interactions between epithelial JAM-A and leukocyte integrins may play a role in regulating PMN TEpM and may be stimulus specific, as previously reported $(37,43,48)$.

In conclusion, we describe a unique murine in vivo pcLoop model that provides new opportunities for analyses of leukocyte migration or responses to a variety of stimuli encompassing specific chemokines, small molecules/drugs, and microbes. Use of the pcLoop for studies of acute mucosal inflammation driven by enteric pathogens is particularly attractive but presents additional challenges that include contributions of direct effects of pathogens on epithelial TJ protein expression/localization/barrier function, as previously reported (49-52). Furthermore, other in vivo models associated with increased PMN TEpM require many hours before measurable TEpM occurs. The acute salmonella colitis model, for example, requires antibiotic pretreatment, and the first appearance of PMN is not until 12-18 hours. The pcLoop model has the advantage of measurable PMN TEpM occurring within 1 hour. The pcLoop model is, thus, an important new tool that may help provide insights into clinically relevant tissue-targeted therapies directed at ameliorating dysregulated leukocyte migration, as seen in many mucosal inflammatory disorders.

\section{Methods}

Animals. Experimental studies were conducted in C57BL/6 WT mice, JAM-A-total KO mice (F11 $1 r^{-1}$ or Jam- $\left.a^{--}\right)$or epithelial-targeted JAM-A-deficient mice (Villin-cre; Jam- $\left.a^{A / f f}\right)$ and littermate controls (Jam-a $\left.a^{A / f 1}\right)$ (as previously described; refs. 38, 53). Villin-cre; Jam- $a^{A / f}$ mice were generated by crossing Jam-a $a^{A / f l}$ mice with a Villin-cre transgenic mouse to obtain intestinal epithelial-specific loss of JAM-A driven by Villin promoter (Supplemental Figure 2). B6.129P2(Cg)-Cdh1tm1Cle/J (E-cadherin-mCFP) mice and LysM-eGFP mice were purchased from the Jackson Laboratory. Animals (8-12 weeks, both sexes) were maintained under standard conditions with 12-hour day/night cycle and access to food and water ad libitum. While Villin-cre; Jam-a $a^{\text {t/f }}$ mice and littermate controls were housed together, fecal transfer was performed between Jam- $a^{+/+}$and Jam- $a^{-/-}$mice.

PcLoop model. Animals were pretreated with proinflammatory cytokines administered by i.p. injection for 24 hours (day 0): TNF- $\alpha$ (100 ng; Peprotech, catalog 315-01A) and IFN- $\gamma$ (100 ng; Peprotech, catalog 31505) in $200 \mu 1$ sterile PBS plus calcium and magnesium solution (PBS plus; Corning Cellgro, Mediatech Inc.). 
At day 1, animals were anesthetized with isoflurane (Fluriso, VETONE) at a constant rate using a rodent anesthesia vaporizer machine (E-Z Anesthesia 7000) and placed on a controlled temperature heat pad to avoid hypothermia. After disinfection of the abdominal skin (isopropyl alcohol 70\%; BD Biosciences alcohol swabs), laparotomy was performed by midline incision. Caecum, terminal ileum and proximal colon were mobilized and exteriorized without disruption of the mesocolon to avoid rupture of the blood supply. Two incisions were made in the mesocolon (in a vessel free area) to isolate a 2-cm length proximal colon segment (pcLoop): 1 incision at $0.5 \mathrm{~cm}$ from the ileo-caecal junction, and the second $2 \mathrm{~cm}$ further distal. A $2 \mathrm{~cm}$ length pcLoop was gently flushed with warm HBSS plus calcium and magnesium (HBSS plus; Corning Cellgro) to remove fecal contents. Flushing the isolated loop was necessary to prevent interference of fecal contents with the even dispersion of chemotactic stimuli across the length of the isolated segment. Furthermore, gentle clearing of luminal contents facilitated normalization of volume of contents to allow for comparative analyses between groups, as well as an accurate quantification of leukocytes by flow cytometry. The 4 generated cut-ends were closed by ligations using nonabsorbable silk suture 3.0 (Braintree Scientific). To induce the recruitment of PMN in the pcLoop lumen, $200 \mu$ sterile HBSS plus containing $1 \mathrm{nM}$ leukotriene $\mathrm{B}_{4}$ (Cayman Chemical) or $1 \mu \mathrm{M}$ chemotactic peptide, fMLF (MilliporeSigma) was injected intraluminally using a 30-gauge needle (30 G 1/2; Becton Dickinson) $(t=0)$. As indicated, 30 minutes prior to administration of chemoattractant, $50 \mu \mathrm{g}$ of function blocking monoclonal antibodies against CD11b/CD18 (clone M1/70, eBioscience, 16-0112-82) or isotype control antibodies (eBioscience, 14-4031-85) were injected in the lumen of pcLoop. The pcLoop was reinserted in the abdominal cavity; then, peritoneum and skin were closed with continuous 2 layer sutures (silk suture 3/8 circle reverse cutting 3-0; Braintree Scientific). At the end of the experiment $(t=60 \mathrm{~min}$ ), the abdomen was reopened to excise the pcLoop. In addition, blood was collected by cardiac puncture prior to euthanasia of the animals by cervical dislocation.

Staining of PMNs in the pcLoop luminal content. Luminal contents of pcLoops were collected followed by flushing with $500 \mu \mathrm{l}$ cold PBS without calcium and magnesium solution (PBS minus; Corning Cellgro) containing 2 mM EDTA (AccuGENE EDTA solution; Lonza). PBS minus solution (500 $\mu$ l) containing $5 \mathrm{mM}$ 1,4-dithiothreitol (DTT; Fisher BioReagents) was added to decrease viscosity caused by mucus. Luminal exudates were filtered through a $35-\mu \mathrm{m}$ nylon mesh cell strainer and collected in a $5 \mathrm{ml}$ round-bottom tube (Falcon, catalog 352235) to remove particulates. Luminal contents were centrifuged (400 $\mathrm{g}$ for 5 min at $4^{\circ} \mathrm{C}$ ), incubated with anti-mouse Fc block (CD16/CD32; BD Bioscience, catalog 553142), and stained with the following fluorochrome-conjugated antibody solution in FACs buffer ( $2 \%$ FBS in PBS minus) for 30 minutes at $4^{\circ} \mathrm{C}$ : anti-CD45-PerCP (clone 30-F11, BD Bioscience, 557235), anti-CD11b-PE (clone M1/70, eBioscience, 12-0112-83), and anti-Ly-6G-Alexa Fluor 647 (clone RB6-8C5, Invitrogen, RM3021). To stain PMN in blood samples, $750 \mu 1$ blood per animal was collected by cardiac puncture and centrifuged ( $400 \mathrm{~g}$ for 10 minutes at $4^{\circ} \mathrm{C}$ ). Serum was removed and erythrocytes were lysed with ammonium chloride potassium solution (ACK Lysing Buffer; Lonza). After incubation with anti-mouse Fc block, a mixture of fluorochrome-conjugated antibody staining was added in FACs buffer for 30 minutes at $4^{\circ} \mathrm{C}$ : anti-CD45-PerCP, anti-CD11b-PE, and anti-Ly-6G-Alexa Fluor 647. Samples were washed and fixed with $4 \%$ paraformaldehyde in PBS prior to flow cytometry analysis. Fluorescent counting beads $(50 \mu 1$, Thermo Fisher Scientific, C36950) was added to samples and quantified via flow cytometry.

Flow cytometric quantification of neutrophils. Quantification of transmigrated PMNs was performed using a NovoCyte Flow Cytometer (ACEA Bioscience). Analyses of flow cytometry data were performed with FlowJo. Cell doublets were excluded by gating FSC area (FSC-A) versus FSC height (FSC-H). PMNs were gated for $\mathrm{CD}_{4} 5^{+}$events and for the expression of Ly-6G/Gr-1 and CD11b. The absolute number of PMNs was determined by using fluorescent counting beads, as recommended by the manufacturer (CountBright, Invitrogen). Results are presented as number of PMN in the pcLoop lumen or number of PMNs per $2 \times 10^{6}$ cells in the epithelial-enriched fraction and LP-enriched fraction.

Collection of epithelial and LP fractions by enzymatic digestion. PcLoops were opened longitudinally, transferred to $15 \mathrm{ml}$ falcon tubes containing PBS minus supplemented with $5 \mathrm{mMDTT}$ (Fisher BioReagents) and 2\% FBS, and washed for 20 minutes at $37^{\circ} \mathrm{C}$ under mechanical agitation at $200 \mathrm{rpm}$. Epithelial cells were collected in $10 \mathrm{ml}$ solution of PBS minus containing $5 \mathrm{mM}$ EDTA and 2\% FBS by mechanical agitation (200 rpm) for 10 minutes at $37^{\circ} \mathrm{C}$ (this step is repeated 3 times). After pooling the enriched epithelial fractions ( $\left.30 \mathrm{ml}\right)$, cells were centrifuged ( $400 \mathrm{~g}$ for $10 \mathrm{~min}$ at $4^{\circ} \mathrm{C}$ ) and washed with ice-cold PBS minus. For the LP-enriched fractions, remaining pcLoops were cut into small pieces $(1-2 \mathrm{~mm}$ ) and digested using RPMI cell media (corning) supplemented with Liberase TM $(2.5 \mathrm{mg} / \mathrm{ml}$; Roche Diagnostics $)$ and DNAse I $\left(2 \times 10^{4}\right.$ Kuntz units $/ \mathrm{ml}$; 
MilliporeSigma) for 30 minutes at $37^{\circ} \mathrm{C}$ under mechanical agitation at $200 \mathrm{rpm}$. Cell suspension was filtered through a $70-\mu \mathrm{m}$ cell strainer and washed in a solution of PBS minus supplemented with $10 \%$ FBS and with 2 mM EDTA. After centrifugation ( $400 \mathrm{~g}$ for 10 minutes at $4^{\circ} \mathrm{C}$ ), cell suspensions were washed with ice-cold PBS minus. Cells from epithelial and LP-enriched fractions were counted using a Scepter 2.0 Cell Counter (MilliporeSigma). Cells $\left(2 \times 10^{6}\right)$ were stained for detection of PMNs by flow cytometry as described above using a mixture of anti-CD45-PerCP, anti-CD11b-PE, and anti-Ly-6G-Alexa Fluor 647. To determine the purity of epithelium- and LP-enriched fractions, B6.129P2(Cg)-Cdh1tm1Cle/J (E-cadherin-mCFP) mice that are characterized by epithelial cells expressing E-cadherin tagged with cyan fluorescent protein were used (Figure 1D). LP- and epithelium-enriched fractions from E-cadherin-mCFP were costained with primary antibodies against EpCAM (anti-EpCAM, clone EGP40/1110; Abcam, catalog ab212580) followed by fluorescent conjugated secondary antibodies (APC-conjugated goat anti-mouse antibody).

Permeability assay. Intestinal permeability was measured by injection of FITC conjugated $4 \mathrm{kDa}$ dextran (MilliporeSigma) at the concentration $1 \mathrm{mg} / \mathrm{ml}$ in $200 \mu \mathrm{HBSS}$ plus into the colonic lumen. For all permeability experiments, FITC-dextran was administrated in the same time as the chemoattractant LTB $_{4}$ in the pcLoop. At the end of the experiment $(t=60 \mathrm{~min})$, blood was collected by cardiac puncture. Fluorescence intensity in the serum was measured with a fluorescent microtiter plate reader (FLUOstar; BMG Labtech). Intact vascular barrier function after pretreatment of animals with $100 \mathrm{ng}$ TNF- $\alpha$ and IFN- $\gamma$ was confirmed as described previously (54). Twenty-four hours after systemic cytokine treatment, $70 \mathrm{kDa}$ Rhodamine-Conjugated Dextran (MilliporeSigma) was injected i.v. via tail vein, and fluorescent digital images of colonic vessels were taken using the Leica Inverted SP5X Confocal Microscope System. Intravascular and interstitial areas near the colonic vessels were selected to evaluate intra- and extravascular optical intensity.

$I H C$. Entire lengths of pcLoop colons were paraformaldehyde-fixed and paraffin-embedded and were then sectioned $(5 \mu \mathrm{m})$ on glass slides. After deparaffinization in xylene and hydration with alcohol solutions, antigen retrieval was performed with Antigen Unmasking Solution (1:100, Vector laboratories, catalog H-3301). Tissue was permeabilized with $0.5 \%$ Triton X-100 (MilliporeSigma) for 10 minutes at room temperature (RT), and endogenous peroxidase activity was abolished with Bloxall solution (Vector laboratories, catalog SP-6000) for 10 minutes at RT. Then, tissue was incubated for 60 minutes with anti-mouse Ig reagent (M.O.M., Vector Laboratories, MPX-2404). After washing with PBS, nonspecific binding sites were blocked with goat serum $2.5 \%$ (Vector laboratories, catalog MP-7444) for 60 minutes at RT. Primary antibody rat anti-mouse Ly-6G (Gr-1) (1:50; clone RB6-8C5, eBioscience, 14-5931-82) was incubated in a humidity chamber at $4^{\circ} \mathrm{C}$ overnight. After washing with PBS plus containing $0.05 \%$ Tween, tissue was incubated with peroxidase-conjugated secondary anti-rat antibody (ImmPRESS HRP anti-rat IgG, Vector Laboratories, MP-7444) for 30 minutes. The bound complexes were visualized by using 3, 3-diaminobenzidine as a substrate (ImmPACT DAB Peroxidase [HRP] Substrate, Vector Laboratories, catalog SK-4105). Identical incubation times were used to enable accurate comparison between all samples. Sections were counterstained with hematoxylin (Hematoxylin QS, Vector laboratories, catalog H-3404) and mounted (Permount; Thermo Fisher Scientific).

Immunofluorescence staining. OCT-embedded sections $(8 \mu \mathrm{m})$ of colon tissue were fixed/permeabilized in absolute ethanol at $-20^{\circ} \mathrm{C}$ for 20 minutes, blocked in PBS plus containing $3 \%$ donkey serum for 1 hour at RT, and incubated with primary antibody goat anti-mouse JAM-A (1:100; R\&D Systems, AF1077) overnight, followed by incubation with labeled secondary antibody (Alexa Fluor 488 donkey anti-goat antibody, Invitrogen) for 1 hour at RT. To visualize cell nuclei, sections were incubated with Hoechst (Invitrogen) for 10 minutes at RT. Paraffin-embedded pcLoop tissues were deparaffinized and rehydrated. Antigen retrieval was performed using citrate buffer $\mathrm{pH} 6$ in a pressure cooker. Tissues were permeabilized with $0.5 \%$ Triton X-100/PBS then stained for PMN (Ly6G/Gr1-Alexa 488, clone RB6-8C5, eBioscience, 14-5931-82) and E-cadherin (1:100; R\&D systems, AF748) in PBS containing 3\% donkey serum. E-cadherin was detected using Alexa-Fluor 555 donkey anti-goat antibody (Invitrogen) and nuclei by using DAPI (Invitrogen). Immunofluorescence images were taken on a confocal microscope (Leica SP5 Inverted 2-Photon FLIM Confocal). ImageJ (NIH) was used for image processing.

Statistics. Results are expressed as means \pm SEM, with each dot representing individual animals. All data represent results from at least 2 independent experiments. Statistical analyses were performed with Prism software (GraphPad Sotfware Inc.). For parametric parameters, the differences were assessed with 2-tailed Student's $t$ test. For nonparametric data, Kruskal-Wallis following Dunn's multiple comparison test, 1-way ANOVA followed by Bonferroni's post-hoc multiple comparison test, or Mann-Whitney $U$ test were used as appropriate. $P<0.05$ was considered significant. 
Study approval. All experimental procedures involving animals were conducted in accordance with the NIH guidelines and protocols approved by the University Committee on Use and Care of Animals at University of Michigan.

\section{Author contributions}

SF and ACL designed the study, performed data collection and data analysis/interpretation, and wrote the manuscript. AN and CAP supervised the study and provided assistance in writing the manuscript.

\section{Acknowledgments}

The authors thank Roland Hilgarth for technical assistance in providing genotyping data on Villin-cre; $J a m-a^{f / f l}$ and Pamela Lincoln for the management of the mouse colonies. This work was supported by German Research Foundation/DFG (FL 870/1-1) to SF, NIH DK59888 and DK55679 to AN, and DK072564, DK079392, and DK061379 to CAP.

Address correspondence to: Charles A Parkos, University of Michigan Medical School, Department of Pathology, NCRC Building 35, Room 30-1537, 2800 Plymouth Road, Ann Arbor, Michigan 48109-2800, USA. Phone: 734.763.6384; Email: cparkos@med.umich.edu.

1. Baumgart DC, Sandborn WJ. Crohn's disease. Lancet. 2012;380(9853):1590-1605.

2. Ordás I, Eckmann L, Talamini M, Baumgart DC, Sandborn WJ. Ulcerative colitis. Lancet. 2012;380(9853):1606-1619.

3. Nourshargh S, Alon R. Leukocyte migration into inflamed tissues. Immunity. 2014;41(5):694-707.

4. Parkos CA. Neutrophil-Epithelial Interactions: A Double-Edged Sword. Am J Pathol. 2016;186(6):1404-1416.

5. Parkos CA, Delp C, Arnaout MA, Madara JL. Neutrophil migration across a cultured intestinal epithelium. Dependence on a CD11b/CD18-mediated event and enhanced efficiency in physiological direction. J Clin Invest. 1991;88(5):1605-1612.

6. Liu Y, Merlin D, Burst SL, Pochet M, Madara JL, Parkos CA. The role of CD47 in neutrophil transmigration. Increased rate of migration correlates with increased cell surface expression of CD47. J Biol Chem. 2001;276(43):40156-40166.

7. Parkos CA, et al. CD47 mediates post-adhesive events required for neutrophil migration across polarized intestinal epithelia. J Cell Biol. 1996;132(3):437-450

8. Zen K, et al. Neutrophil migration across tight junctions is mediated by adhesive interactions between epithelial coxsackie and adenovirus receptor and a junctional adhesion molecule-like protein on neutrophils. Mol Biol Cell. 2005;16(6):2694-2703.

9. Ebnet K. Junctional Adhesion Molecules (JAMs): Cell Adhesion Receptors With Pleiotropic Functions in Cell Physiology and Development. Physiol Rev. 2017;97(4):1529-1554

10. Liu Y, et al. Human junction adhesion molecule regulates tight junction resealing in epithelia. J Cell Sci. 2000;113(Pt 13):2363-2374.

11. Luissint AC, Nusrat A, Parkos CA. JAM-related proteins in mucosal homeostasis and inflammation. Semin Immunopathol. 2014;36(2):211-226.

12. Martìn-Padura I, et al. Junctional adhesion molecule, a novel member of the immunoglobulin superfamily that distributes at intercellular junctions and modulates monocyte transmigration. J Cell Biol. 1998;142(1):117-127.

13. Jiminez JA, Uwiera TC, Douglas Inglis G, Uwiera RR. Animal models to study acute and chronic intestinal inflammation in mammals. Gut Pathog. 2015;7:29.

14. Kiesler P, Fuss IJ, Strober W. Experimental Models of Inflammatory Bowel Diseases. Cell Mol Gastroenterol Hepatol. 2015;1(2):154-170.

15. Valatas V, Vakas M, Kolios G. The value of experimental models of colitis in predicting efficacy of biological therapies for inflammatory bowel diseases. Am J Physiol Gastrointest Liver Physiol. 2013;305(11):G763-G785.

16. Arques JL, et al. Salmonella induces flagellin- and MyD88-dependent migration of bacteria-capturing dendritic cells into the gut lumen. Gastroenterology. 2009;137(2):579-587.

17. Edelblum KL, et al. $\gamma \delta$ Intraepithelial Lymphocyte Migration Limits Transepithelial Pathogen Invasion and Systemic Disease in Mice. Gastroenterology. 2015;148(7):1417-1426.

18. Germain RN, Robey EA, Cahalan MD. A decade of imaging cellular motility and interaction dynamics in the immune system. Science. 2012;336(6089):1676-1681.

19. Hashimoto S, et al. Intravital imaging of neutrophil recruitment in intestinal ischemia-reperfusion injury. Biochem Biophys Res Commun. 2018;495(3):2296-2302.

20. Hoytema van Konijnenburg DP, Reis BS, Pedicord VA, Farache J, Victora GD, Mucida D. Intestinal Epithelial and Intraepithelial T Cell Crosstalk Mediates a Dynamic Response to Infection. Cell. 2017;171(4):783-794.e13.

21. Secklehner J, Lo Celso C, Carlin LM. Intravital microscopy in historic and contemporary immunology. Immunol Cell Biol. 2017;95(6):506-513.

22. Toiyama Y, et al. Intravital imaging of DSS-induced cecal mucosal damage in GFP-transgenic mice using two-photon microscopy. J Gastroenterol. 2010;45(5):544-553.

23. Faust N, Varas F, Kelly LM, Heck S, Graf T. Insertion of enhanced green fluorescent protein into the lysozyme gene creates mice with green fluorescent granulocytes and macrophages. Blood. 2000;96(2):719-726.

24. Afonso PV, et al. LTB4 is a signal-relay molecule during neutrophil chemotaxis. Dev Cell. 2012;22(5):1079-1091.

25. Chen M, et al. Neutrophil-derived leukotriene B4 is required for inflammatory arthritis. J Exp Med. 2006;203(4):837-842.

26. Chou RC, et al. Lipid-cytokine-chemokine cascade drives neutrophil recruitment in a murine model of inflammatory arthritis. 
Immunity. 2010;33(2):266-278.

27. Lämmermann T, et al. Neutrophil swarms require LTB4 and integrins at sites of cell death in vivo. Nature. 2013;498(7454):371-375.

28. Oyoshi MK, et al. Leukotriene B4-driven neutrophil recruitment to the skin is essential for allergic skin inflammation. Immunity. 2012;37(4):747-758.

29. Satpathy SR, et al. Crystalline silica-induced leukotriene B4-dependent inflammation promotes lung tumour growth. Nat Commun. 2015;6:7064.

30. Bruewer M, et al. Proinflammatory cytokines disrupt epithelial barrier function by apoptosis-independent mechanisms. $J$ Immunol. 2003;171(11):6164-6172.

31. Garlanda C, Dejana E. Heterogeneity of endothelial cells. Specific markers. Arterioscler Thromb Vasc Biol. 1997;17(7):1193-1202.

32. Sumagin R, et al. Neutrophil interactions with epithelial-expressed ICAM-1 enhances intestinal mucosal wound healing. Mucosal Immunol. 2016;9(5):1151-1162.

33. van Buul JD, Kanters E, Hordijk PL. Endothelial signaling by Ig-like cell adhesion molecules. Arterioscler Thromb Vasc Biol. 2007;27(9):1870-1876

34. Luissint AC, Parkos CA, Nusrat A. Inflammation and the Intestinal Barrier: Leukocyte-Epithelial Cell Interactions, Cell Junction Remodeling, and Mucosal Repair. Gastroenterology. 2016;151(4):616-632.

35. Monteiro AC, Parkos CA. Intracellular mediators of JAM-A-dependent epithelial barrier function. Ann N Y Acad Sci. 2012;1257:115-124.

36. Ebnet K, Suzuki A, Ohno S, Vestweber D. Junctional adhesion molecules (JAMs): more molecules with dual functions? J Cell Sci. 2004;117(Pt 1):19-29.

37. Woodfin A, et al. JAM-A mediates neutrophil transmigration in a stimulus-specific manner in vivo: evidence for sequential roles for JAM-A and PECAM-1 in neutrophil transmigration. Blood. 2007;110(6):1848-1856.

38. Laukoetter MG, et al. JAM-A regulates permeability and inflammation in the intestine in vivo. J Exp Med. 2007;204(13):3067-3076.

39. Marchiando AM, et al. Caveolin-1-dependent occludin endocytosis is required for TNF-induced tight junction regulation in vivo. J Cell Biol. 2010;189(1):111-126.

40. Coombes BK, et al. Analysis of the contribution of Salmonella pathogenicity islands 1 and 2 to enteric disease progression using a novel bovine ileal loop model and a murine model of infectious enterocolitis. Infect Immun. 2005;73(11):7161-7169.

41. Everest P, et al. Evaluation of Salmonella typhimurium mutants in a model of experimental gastroenteritis. Infect Immun. 1999;67(6):2815-2821

42. Zen K, Liu Y, Cairo D, Parkos CA. CD11b/CD18-dependent interactions of neutrophils with intestinal epithelium are mediated by fucosylated proteoglycans. J Immunol. 2002;169(9):5270-5278.

43. Ostermann G, Weber KS, Zernecke A, Schröder A, Weber C. JAM-1 is a ligand of the beta(2) integrin LFA-1 involved in transendothelial migration of leukocytes. Nat Immunol. 2002;3(2):151-158.

44. Wojcikiewicz EP, et al. LFA-1 binding destabilizes the JAM-A homophilic interaction during leukocyte transmigration. Biophys J. 2009;96(1):285-293.

45. Brazil JC, Parkos CA. Pathobiology of neutrophil-epithelial interactions. Immunol Rev. 2016;273(1):94-111.

46. Cera MR, et al. JAM-A promotes neutrophil chemotaxis by controlling integrin internalization and recycling. J Cell Sci. 2009;122(Pt 2):268-277.

47. Corada M, et al. Junctional adhesion molecule-A-deficient polymorphonuclear cells show reduced diapedesis in peritonitis and heart ischemia-reperfusion injury. Proc Natl Acad Sci USA. 2005;102(30):10634-10639.

48. Shaw SK, et al. Coordinated redistribution of leukocyte LFA-1 and endothelial cell ICAM-1 accompany neutrophil transmigration. J Exp Med. 2004;200(12):1571-1580.

49. Guttman JA, Li Y, Wickham ME, Deng W, Vogl AW, Finlay BB. Attaching and effacing pathogen-induced tight junction disruption in vivo. Cell Microbiol. 2006;8(4):634-645.

50. Nusrat A, von Eichel-Streiber C, Turner JR, Verkade P, Madara JL, Parkos CA. Clostridium difficile toxins disrupt epithelial barrier function by altering membrane microdomain localization of tight junction proteins. Infect Immun. 2001;69(3):1329-1336

51. Sakaguchi T, Köhler H, Gu X, McCormick BA, Reinecker HC. Shigella flexneri regulates tight junction-associated proteins in human intestinal epithelial cells. Cell Microbiol. 2002;4(6):367-381.

52. Sonoda N, et al. Clostridium perfringens enterotoxin fragment removes specific claudins from tight junction strands: Evidence for direct involvement of claudins in tight junction barrier. J Cell Biol. 1999;147(1):195-204.

53. Rahman K, et al. Loss of Junctional Adhesion Molecule A Promotes Severe Steatohepatitis in Mice on a Diet High in Saturated Fat, Fructose, and Cholesterol. Gastroenterology. 2016;151(4):733-746.e12.

54. Flemming S, Burkard N, Meir M, Schick MA, Germer CT, Schlegel N. Sphingosine-1-Phosphate Receptor-1 Agonist Sew2871 Causes Severe Cardiac Side Effects and Does Not Improve Microvascular Barrier Breakdown in Sepsis. Shock. 2018;49(1):71-81. 